\title{
Continuum theory of bending-to-stretching transition
}

\author{
O. U. Salman $\odot,{ }^{1}$ G. Vitale, ${ }^{2}$ and L. Truskinovsky ${ }^{3}$ \\ ${ }^{1}$ CNRS, LSPM UPR3407, Université Paris 13, Sorbonne Paris Cité, 93430 Villetaneuse, France \\ ${ }^{2}$ Laboratoire de Mécanique des Solides, CNRS-UMR 7649, Ecole Polytechnique, Route de Saclay, F-91128 Palaiseau Cedex, France \\ ${ }^{3}$ PMMH, CNRS-UMR 7636 PSL-ESPCI, 10 Rue Vauquelin, 75005 Paris, France
}

(Received 2 June 2019; published 6 November 2019)

\begin{abstract}
Transition from bending-dominated to stretching-dominated elastic response in semiflexible fibrous networks plays an important role in the mechanical behavior of cells and tissues. It is induced by changes in network connectivity and relies on the construction of new cross-links. We propose a simple continuum model of this transition with macroscopic strain playing the role of order parameter. An unusual feature of this Landau-type theory is that it is based on a single-well potential. The theory predicts that bending-to-stretching transition should proceed through propagation of the fronts separating domains with affine and nonaffine elastic response.
\end{abstract}

DOI: 10.1103/PhysRevE.100.051001

Typical force transmitting systems in cellular biology can be viewed as networks of cross-linked semiflexible fibers which respond to mechanical loading by both stretching and bending [1-6]. One of the most striking features of such "materials" is the loading-induced transition from nonaffine, bending-dominated elasticity, to (almost) affine, stretchingdominated elasticity [7-9]. This transition is accompanied by the anomalous growth of elastic moduli and is usually linked to the increase of the cross-linker density [10].

In highly connected dense networks the stretching stiffness dominates because they cannot be deformed without either elongation or shortening of the links; in less dense, underconstrained networks, classical rigidity is lost due to the appearance of floppy modes and softer, bending elasticity becomes responsible for the overall stiffness [11-13]. The bendingto-stretching (BS) transition was successfully simulated in two-dimensional and three-dimensional athermal microscopic models. In particular, it was found that a continuous crossover between the two regimes takes the form of a highly heterogeneous coexistence between bending- (B) and stretching- (S) dominated phases [1-3,7-9].

Despite these successes in microscale modeling, the fundamental understanding of the BS transition at the macroscopic level is still lacking. The development of a coarse-grained model of this phenomenon will facilitate the continuum modeling of cellular scale phenomena [14-18] and advance the design of the artificial metamaterials with underconnected network architecture [5,19,20].

In this Rapid Communication we develop a prototypical Landau-type theory of the strain-induced BS transition. We build on the idea that cross-linked networks have the ability to internally rearrange in response to the applied deformation through fiber rotation $[3,21,22]$ and that new cross-links can form in this process [6,23]. Our main result is the regime diagram showing how the dominating deformation mode is controlled by the applied strain and the dimensionless ratio of the internal and external length scales.

Our approach is deliberately minimalistic. As a prototype of a semiflexible network, we use a pantographic structure with freely rotating cross-links, as in a collapsible arm of wall mounted mirror [24]. The crucial assumption is that this floppy mechanical system can be stabilized by breakable springs whose role is to ensure rigidity when they are intact (see Fig. 1). This simple structure is chosen as an archetype of a broad class of semiflexible networks studied previously by direct numerical approaches.

Suppose that the initial state of the structure shown in Fig. 1 is chosen in such a way that all vertical springs are disengaged and the system is underconstrained [25,26]. If such structure is stretched, the geometrical constraints force the system to contract in the vertical direction, which can lead to the rebuilding of the bonds. As a result, an underconstrained system transforms into an overconstrained one.

We assume that the floppy pantographic structure itself is built of inextensible but flexible beams connected through pivots. It is known that the macroscopic elastic response of such structure is B dominated [27]; more complex examples of B-dominated structures can be found in the theory of high contrast elastic composites [28-30]. In the continuum representation of such systems the nonlocal (higher order) elasticity appears already at the leading order in the homogenization limit, which leads to elasticity theories dominated by an internal length scale (as in liquid crystals [31]).

Therefore, we can model the discrete structure shown in Fig. 1 as a one-dimensional (1D) continuum whose classical elastic energy "softens" in compression due to breaking of the reinforcing springs. An additive quadratic strain gradient term in the energy density can be then used as a proxy for the higher order (nonclassical) elasticity of the pantographic frame. With macroscopic strain playing the role of the order parameter [32], the ensuing model takes the form of a Ginzburg-Landau (GL) theory [33]. It is characterized, however, by an unusual (vertically) flipped Lennard-Jones- type potential (see Fig. 2).

We use this continuum model to show that the quasistatically driven BS transition proceeds through nucleation and propagation of the fronts separating domains with affine and nonaffine elastic response. At such fronts, the connectivity of the network changes and they can be interpreted as the 


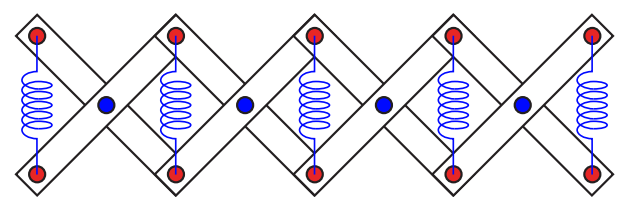

FIG. 1. Floppy pantographic structure reinforced by vertical "breakable" bonds.

(degenerate) domain walls. In contrast to the mixed BS states, the pure B and $\mathrm{S}$ states are homogeneous. We show, however, that in the B states the affinity of the deformation, imposed by the weak gradient elasticity, is not robust.

To mimic realistic systems we also consider the GL model with a constraining elastic background [34], either imitating the surrounding matrix [35] or representing nonmechanical long-range signaling [35-37]. The resultant competing interactions generate in the mixed $\mathrm{BS}$ regime stable periodic patterns reminiscent of what is observed in other reinforced fragile systems [38-40].

We write the coarse-grained (homogenized) dimensionless energy of the system shown in Fig. 1 in the form $F=$ $\int_{0}^{1} f\left(\varepsilon, \varepsilon^{\prime}\right) d x$, where $\varepsilon(x)=u^{\prime}(x)$ is the longitudinal strain, $u(x)$ is the displacement of point $x$, and the prime denotes derivative. With our focus on macroscale properties we surpass the scale of individual subelements (beams and pivots) and, in contrast to more detailed studies, e.g., [5], target only the parameters which vary slowly at the scale of the microstructure.

Since at each macroscopic point our model should account for two main elements, breakable springs and pantographic matrix, we assume that the energy density has an additive structure

$$
f\left(\varepsilon, \varepsilon^{\prime}\right)=f_{1}(\varepsilon)+f_{2}\left(\varepsilon^{\prime}\right) .
$$

The first term in (1), accounting for breakable springs, is a single-well potential. It should capture sound elasticity at sufficient stretching of the structure, when springs are forced (by the structure) to engage. Instead, it should describe softening in response to compression with progressive degeneration of elasticity as springs are forced to disengage. We show in Fig. 2 a particular potential with these properties which we use in computations: $f_{1}(\varepsilon)=\left(\varepsilon_{0}-\varepsilon\right)^{-2}-2\left(\varepsilon_{0}-\right.$ $\varepsilon)^{-1}$; however, our general results are independent of this choice.

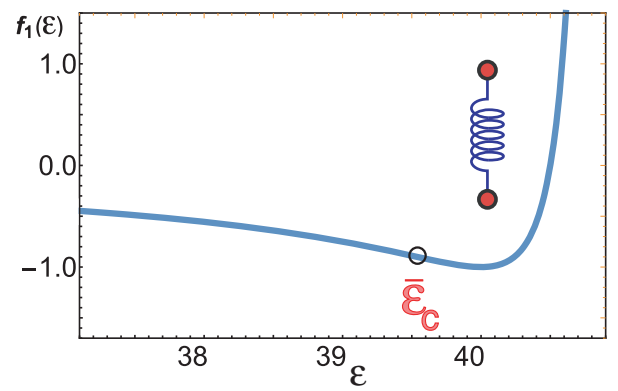

FIG. 2. The flipped Lennard-Jones-type potential $f_{1}$ with $\varepsilon_{0}=$ 41. At the spinodal limit $\partial^{2} f_{1}\left(\bar{\varepsilon}_{c}\right)=0$.
The second term in (1) characterizes the pantographic structure itself. Since it is floppy with respect to affine longitudinal deformations, the homogenized elastic modulus is equal to zero. However, the nonaffine longitudinal deformations remain penalized by the bending of individual beams. Following [27], where rigorous homogenization of the pantographic structure was performed, we describe its macroscale properties by the energy density $f_{2}\left(\varepsilon^{\prime}\right)=\left(\lambda_{b}^{2} / 2\right) \varepsilon^{\prime 2}$, where $\lambda_{b}$ is the internal length scale; despite the fact that we operate with longitudinal strain, this macroscopic energy originates from microscale bending.

We further assume that the system is loaded in the "hard" loading device, which means that the control parameter is the applied strain $\bar{\varepsilon}$, so that, for instance, $u(0)=-\bar{\varepsilon} / 2, u(1)=$ $\bar{\varepsilon} / 2$. We also suppose that the boundaries of our 1D continuum system are "moment free" in the sense that $u^{\prime \prime}(0)=$ $u^{\prime \prime}(1)=0$.

The affine configuration $\bar{u}(x)=(\bar{\varepsilon} / 2)(2 x-1)$ is always an equilibrium state; however, it is not always stable. To find the instability threshold we study a linearized problem involving the displacement perturbation $s(x)=u(x)-\bar{u}(x)$. The problem reduces to finding nontrivial solutions of the linear equation $-\lambda_{b}^{2} s^{\prime \prime \prime \prime}+\partial^{2} f_{1}(\bar{\varepsilon}) s^{\prime \prime}=0$, where $\partial^{2} f_{1}(\bar{\varepsilon})=$ $\partial^{2} f_{1} /\left.\partial \varepsilon^{2}\right|_{\bar{\varepsilon}}$ and the boundary conditions are $s(0)=s(1)=$ $s^{\prime \prime}(0)=s^{\prime \prime}(1)=0$. The nonaffine modes $\sim \sin (n \pi x)$ appear at $\bar{\varepsilon}$ solving the characteristic equation

$$
\partial^{2} f_{1}(\bar{\varepsilon})=-\lambda_{b}^{2}(n \pi)^{2}
$$

When the instability of the homogeneous state is associated with stretching, we need to find $n=n_{c}$ for which the value of $\bar{\varepsilon}$, solving (2), is the smallest. Instead, if the homogeneous state loses stability in compression, we need to find $n=n_{c}$ for which the value of $\bar{\varepsilon}$, solving (2), is the largest.

The analysis of (2) shows that the affine configuration is locally stable for sufficiently small $\bar{\varepsilon} \leqslant \bar{\varepsilon}_{c}^{*}$ and for sufficiently large $\bar{\varepsilon} \geqslant \bar{\varepsilon}_{c}^{* *}$. Both direct (affine to nonaffine) and return (nonaffine to affine) instabilities are of long wave nature with the same critical wavelength $n_{c}=1$. Note that $\bar{\varepsilon}_{c}^{*} \leqslant \bar{\varepsilon}_{c}^{* *} \leqslant \bar{\varepsilon}_{c}$, where $\bar{\varepsilon}_{c}$ is the spinodal limit satisfying $\partial^{2} f_{1}\left(\bar{\varepsilon}_{c}\right)=0$, so the nonaffine configurations are located inside the concavity domain of the potential $f_{1}$. The set of pairs $(n, \bar{\varepsilon})$ solving (2) is shown in Fig. 3(a) by the black squares.

We associate stable affine states at $\bar{\varepsilon} \geqslant \bar{\varepsilon}_{c}^{* *}$ with Sdominated regimes and at $\bar{\varepsilon} \leqslant \bar{\varepsilon}_{c}^{*}$ with B-dominated regimes. In $\mathrm{S}$ regimes the affine character of the deformation is secured by the presence of classical elasticity. The latter becomes
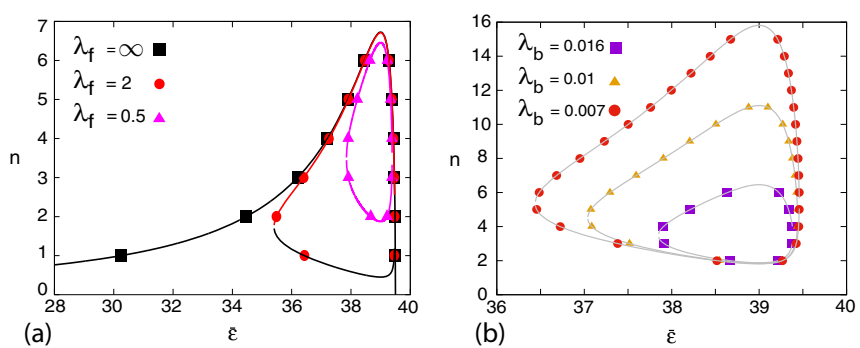

FIG. 3. The pairs $(n, \bar{\varepsilon})$ solving (4): (a) varying parameter $\lambda_{f}$ at fixed $\lambda_{b}=0.0167$; (b) varying parameter $\lambda_{b}$ at fixed $\lambda_{f}=0.35$. Continuous interpolations are added as a guide for the eye. 


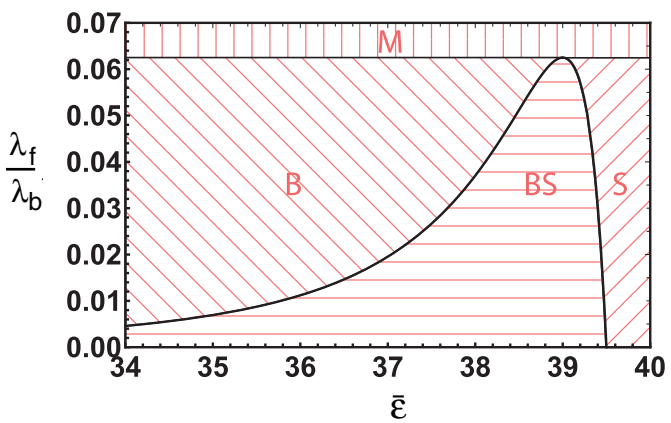

FIG. 4. Regime diagram for the infinite size system: (M) matrixdominated phase; (B) bending-dominated phase; (S) stretchingdominated phase; (BS) mixture phase.

destabilizing in the $\mathrm{B}$ regimes where the affinity is safeguarded by the (nonclassical) gradient elasticity.

To account for nonlocal interactions in the realistic biological systems we now embed our floppy frame into an elastic environment. To this end we introduce linear coupling of our GL system with a prestretched background and consider dimensionless energy [41]

$$
F=\int_{0}^{1}\left\{f\left(\varepsilon, \varepsilon^{\prime}\right)+\left[1 /\left(2 \lambda_{f}^{2}\right)\right][u-\bar{u}(x)]^{2}\right\} d x,
$$

where $\lambda_{f}$ is the external length scale characterizing the relative size of the embedding matrix. Since the order parameter is $u^{\prime}(x)$, the account of environmental elasticity brings implicit nonlocality into the conventional structure of a GL theory [42].

The linear stability condition for the affine state in the model (3) takes the form

$$
\lambda_{b}^{2}(n \pi)^{4}+\partial^{2} f_{1}(\bar{\varepsilon})(n \pi)^{2}+1 / \lambda_{f}^{2}=0 .
$$

One can show that the redressed upper $\bar{\varepsilon}_{c}^{* *}$ and lower $\bar{\varepsilon}_{c}^{*}$ critical strains correspond again to the same critical wavelength; however, $n_{c}$ can now take arbitrary large values. In Fig. 3 we illustrate the dependence of the pairs $(n, \bar{\varepsilon})$ solving (4) on dimensionless lengths $\lambda_{b}$ and $\lambda_{f}$.

The obtained results can be summarized in the form of a regime diagram. For an infinite system $\left(\lambda_{b} \rightarrow \infty, \lambda_{f} \rightarrow \infty\right)$ one can disregard the discreteness of the problem, and write the equation for the critical strain in the form

$$
\partial^{2} f_{1}\left(\bar{\varepsilon}_{c}\right)=2\left(\lambda_{b} / \lambda_{f}\right) .
$$

The solution of (5) can be used as a description of the boundary delineating the pure B and $\mathrm{S}$ regimes from the mixed BS regime. In such regime diagram (see Fig. 4) the applied strain plays the role analogous to the cross-linker density, while the ratio $\lambda_{b} / \lambda_{f}$ characterizes the stabilizing strength of the elastic environment; in the matrix-dominated (supercritical) regime $M$ the deformation is always affine.

To explore the structure of the nonlinear energy minimizing configurations we need to solve the equation

$$
-\lambda_{b}^{2} u^{\prime \prime \prime \prime}+\partial^{2} f_{1}\left(u^{\prime}\right) u^{\prime \prime}-\left(1 / \lambda_{f}^{2}\right)[u-\bar{u}(x)]=0,
$$

for which we used a numerical continuation method [43].

In the limiting case $\lambda_{f}=\infty$ the nonlocality is absent and the energy minimizers have the basic GL structure. Along the
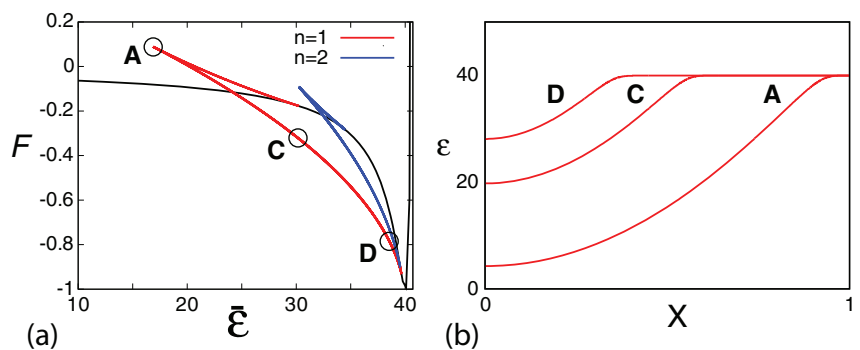

(b)

FIG. 5. Solutions of the nonlinear problem (6) in the case $\lambda_{f}=$ $\infty$ : (a) the equilibrium elastic energy $F(\bar{\varepsilon})$; black line indicates the trivial homogeneous branch; (b) the equilibrium strain profiles in points $A, C$, and $D$ in (a). Here $\lambda_{b}=0.0167$.

energy minimizing path [see Fig. 5(a)], we observe nucleation of a single domain boundary separating the phase where the springs are broken, and the elasticity is of B type, from the phase where they are intact, and the elasticity is of the $\mathrm{S}$ type [see Fig. 5(b)]. Other equilibrium branches, describing more complex mixtures of such phases, have higher energy [see the branch with two interfaces $(n=2)$ in Fig. 5(a)].

Note that despite the familiar GL structure of the theory, the ensuing two-phase configurations are far from being conventional. Consider, for instance, a stretching loading protocol originating in the homogeneous B phase, and assume that the system always remains in the ground state. The nucleation of the $\mathrm{S}$ phase takes place discontinuously with the formation of the configuration $C$ [see Fig. 5(b)] exhibiting a front separating the affine $\mathrm{S}$ phase and the nonaffine $\mathrm{B}$ phase. As the applied strain $\bar{\varepsilon}$ increases, the homogeneous $\mathrm{S}$ phase proliferates with successive springs reconnecting. During this process the shrinking B phase maintains a particular pattern of nonaffinity [see configurations $C$ and $D$ in Fig. 5(b)]. The $\mathrm{S}$ phase finally takes over through a discontinuous event of the final annihilation of the B phase.

Consider now the general case $0<\lambda_{f}<\infty$. The bending energy term favors coarsening while the nonlocal term drives the refinement of the microstructure and the ensuing competition leads to the formation of BS mixtures with more complex geometry. In Fig. 6(a) we show the typical configuration of the low-energy branches. Note that the topological structure of the microconfiguration changes along the global minimum path: we observe a switch from a configuration with four
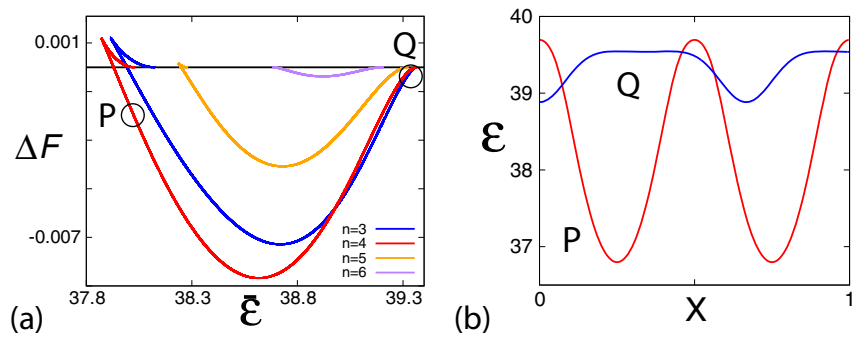

FIG. 6. Lowest-energy branches in the case $0<\lambda_{f}<\infty$; (a) the energy difference between the actual configuration and the homogeneous configuration; (b) the equilibrium strain profiles for the branches with $n=4$ and $n=3$ corresponding to the points $P$ and $Q$ in (a). Parameters: $\lambda_{b}=0.0167$ and $\lambda_{f}=0.45$. 


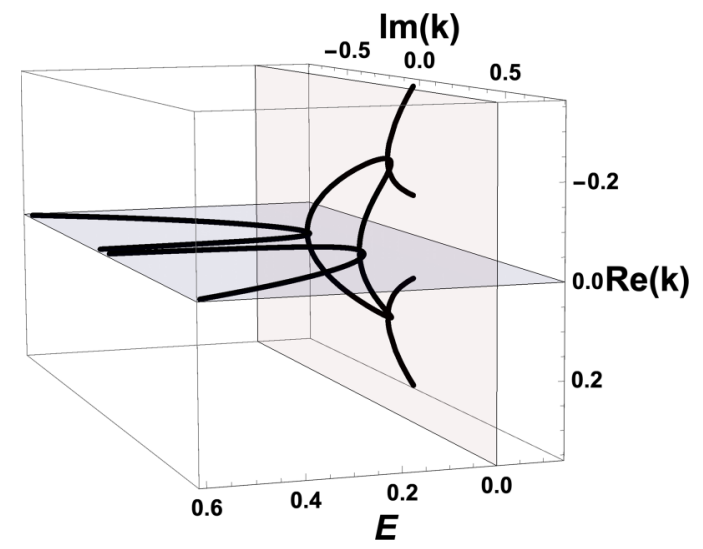

FIG. 7. Complex roots of the characteristic equation when $\lambda_{b} / \lambda_{f}=0.03$. The plane $\operatorname{Re} k=0$ is highlighted by blue.

$(n=4)$ to a configuration with three $(n=3)$ interfaces [see Fig. 6(b)].

As the total strain $\bar{\varepsilon}$ increases beyond the point $P$, the homogeneous B phase loses stability, which leads to collective nucleation of the periodically placed islands of the affine $S$ phase while the remaining B phase becomes nonaffine. With a further increase of $\bar{\varepsilon}$, the islands of S phase grow in size (see point Q) and eventually B phase completely disappears.

We now discuss the fact that in our simple tests the deformation in the pure $\mathrm{S}$ and $\mathrm{B}$ phases was affine; the nonaffine response was observed only in the BS (mixed) phase. We recall that in experiments involving disordered fibrous networks, the nonaffinity of the deformation was found in the whole range of the B-dominated elastic response [5]. These observations can be explained by the fragility of the affine response in B phase while such response is robust in $\mathrm{S}$ phase.

Indeed, consider again the linear modes $\sim \exp (i k x)$ superimposed on a homogeneous solution of (6). The normalized wave numbers $k$ must satisfy the characteristic equation $k^{4}+$ $E k^{2}+\left(\lambda_{b} / \lambda_{f}\right)^{2}=0$, where for convenience we now introduced directly the tangential elastic modulus $E(\bar{\varepsilon})=\partial^{2} f_{1}(\bar{\varepsilon})$. The complex solutions of this equation are shown in Fig. 7. Note that in $\mathrm{S}$ phase the roots $k$ are purely imaginary. They describe exponential decay of the local perturbations and are characteristic for systems with affine response. Instead, in $B$ phase the characteristic wave numbers are real and the perturbations spread over the whole system signaling a nonaffine response. In the crossover range, where $E<0$ and the scales $(-E)^{1 / 2} \lambda_{f}$ and $\left(\lambda_{b} \lambda_{f}\right)^{1 / 2}$ are comparable [see (5) for the more precise characterization], the wave numbers are complex and the response is mixed.

To further support these observations we again linearize (6) but now impose a localized perturbation $g(x)$ :

$$
-\lambda_{b}^{2} u^{\prime \prime \prime \prime}+E u^{\prime \prime}-\left(1 / \lambda_{f}^{2}\right)[u-\bar{u}(x)-g(x)]=0 .
$$

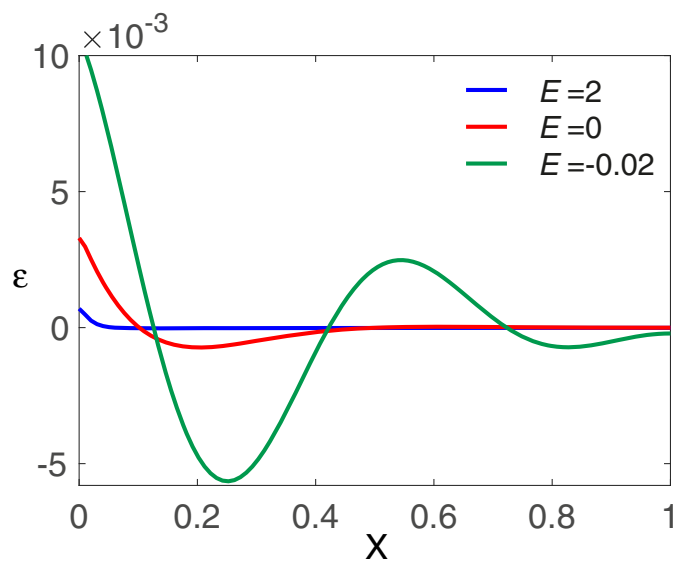

FIG. 8. Strain profiles appearing in response to a localized force distribution described by the function $g(x)=0.1 \operatorname{sech}[150(x-$ $0.001)]^{2}$. Parameters: $\bar{\epsilon}=0, \lambda_{b}=0.0167$, and $\lambda_{f}=0.5$.

The response to such force distribution, when it is applied near one of the ends, is illustrated in Fig. 8. We see an almost unperturbed affine response in the $\mathrm{S}$ phase, a limited penetration of the perturbation in the BS phase, and a markedly nonaffine global response in the $\mathrm{B}$ phase.

To show that these observations are not conditioned to the case with elastic environment, consider a linearized problem for a bar with $\lambda_{f}=\infty$ which is clamped on one side and loaded on the other side by a force $h$. Suppose that the bending rigidity $\lambda_{b}$ is sufficiently small, so that in $S$ phase we can neglect bending and relax the clamping boundary condition. Under these assumptions the problem reduces to solving the equation $u^{\prime \prime}=0$ with the boundary conditions $u(0)=$ $0, E u^{\prime}(1)=h$. The resulting response is affine: $\varepsilon=h / E$. Now consider B phase, where the stiffness $E$ can be neglected and the equilibrium equation is $u^{\prime \prime \prime \prime}=0$, while the boundary conditions are $u(0)=0, u^{\prime}(0)=0, u^{\prime \prime}(1)=0,-\lambda_{b}^{2} u^{\prime \prime \prime}(1)=h$. The solution of this boundary value problem is globally inhomogeneous: $\varepsilon=\left(h / \lambda_{b}^{2}\right) x(1-x / 2)$.

To conclude, we presented a prototypical continuum model of the BS transition. Our simple description, amenable to analytical study, complements recent comprehensive microscopic simulations of this phenomenon. We interpreted BS transition in the framework of the Ginzburg-Landau formalism building in this way a link with the conventional theory of first-order phase transitions. The proposed model provides a theoretical explanation of the markedly no-affine response in the Bdominated regime and rationalizes the observed heterogeneity of the mixed BS phase.

We thank P. Recho and P. Ciarletta for helpful discussions. The work was supported by the grants ANR-18-CE42-0017 (O.U.S.) and ANR-10-IDEX-0001-02 PSL (L.T.).
[1] J. Wilhelm and E. Frey, Phys. Rev. Lett. 91, 108103 (2003).

[2] D. A. Head, A. J. Levine, and F. C. MacKintosh, Phys. Rev. E 68, 061907 (2003).
[3] P. R. Onck, T. Koeman, T. Van Dillen, and E. Van der Giessen, Phys. Rev. Lett. 95, 178102 (2005).

[4] H. Kang, Q. Wen, P. A. Janmey, J. X. Tang, E. Conti, and F. C. MacKintosh, J. Phys. Chem. B 113, 3799 (2009). 
[5] C. P. Broedersz and F. C. MacKintosh, Rev. Mod. Phys. 86, 995 (2014).

[6] A. F. Pegoraro, P. Janmey, and D. A. Weitz, Cold Spring Harb. Perspect. Biol. 9, (2017).

[7] G. A. Buxton and N. Clarke, Phys. Rev. Lett. 98, 238103 (2007).

[8] X. Mao, O. Stenull, and T. C. Lubensky, Phys. Rev. E 87, 042601 (2013).

[9] C. P. Broedersz, M. Sheinman, and F. C. MacKintosh, Phys. Rev. Lett. 108, 078102 (2012).

[10] J. Feng, H. Levine, X. Mao, and L. M. Sander, Soft Matter 12, 1419 (2016).

[11] D. A. Head, A. J. Levine, and F. C. MacKintosh, Phys. Rev. Lett. 91, 108102 (2003).

[12] M. Das, F. C. MacKintosh, and A. J. Levine, Phys. Rev. Lett. 99, 038101 (2007).

[13] C. P. Broedersz, X. Mao, T. C. Lubensky, and F. C. MacKintosh, Nat. Phys. 7, 983 (2011).

[14] F. Meng and E. M. Terentjev, Macromolecules 51, 4660 (2018).

[15] J. Prost, F. Jülicher, and J.-F. Joanny, Nat. Phys. 11, 111 (2015).

[16] A. Bernheim-Groswasser, N. S. Gov, S. A. Safran, and S. Tzlil, Adv. Mater. 30, 1707028 (2018).

[17] B. Burkel, A. Lesman, P. Rosakis, D. A. Tirrell, G. Ravichandran, and J. Notbohm, Mechanics of Biological Systems and Materials (Springer International Publishing, Cham, 2017), Vol. 6, pp. 135-141.

[18] A. Taloni, E. Kardash, O. U. Salman, L. Truskinovsky, S. Zapperi, and C. A. M. La Porta, Phys. Rev. Lett. 114, 208101 (2015).

[19] M. F. Ashby, Philos. Trans. R. Soc., A 364, 15 (2006).

[20] D. Z. Rocklin, S. Zhou, K. Sun, and X. Mao, Nat. Commun. 8, 14201 (2017).

[21] C. Heussinger and E. Frey, Phys. Rev. Lett. 97, 105501 (2006).

[22] Q. Wen and P. A. Janmey, Exp. Cell Res. 319, 2481 (2013).

[23] M. L. Gardel, J. H. Shin, F. C. MacKintosh, L. Mahadevan, P. Matsudaira, and D. A. Weitz, Science 304, 1301 (2004).
[24] E. van der Giessen, Nat. Phys. 7, 923 (2011).

[25] J. C. Maxwell, London, Edinburgh, Dublin Philos. Mag. J. Sci. 27, 294 (1864).

[26] C. R. Calladine, Int. J. Solids Struct. 14, 161 (1978).

[27] J.-J. Alibert, P. Seppecher, and F. Dell'Isola, Math. Mech. Solids 8, 51 (2003).

[28] K. D. Cherednichenko, V. P. Smyshlyaev, and V. V. Zhikov, Proc. R. Soc. Edinburgh, Sec. A: Math. 136, (2006).

[29] C. Boutin, J. Soubestre, M. S. Dietz, and C. Taylor, Eur. J. Mech. A: Solids 42 (2013).

[30] M. Camar-Eddine and P. Seppecher, Arch. Ration. Mech. Anal. 170, 211 (2003).

[31] P. M. Chaikin, T. C. Lubensky, and T. A. Witten, Principles of Condensed Matter Physics (Cambridge University Press, Cambridge, 1995), Vol. 1.

[32] L. Golubović and T. C. Lubensky, Phys. Rev. Lett. 63, 1082 (1989).

[33] L. Truskinovsky, Contemporary Research in the Mechanics and Mathematics of Materials, Barcelona (CIMNE, Barcelona, 1996), pp. 322-332.

[34] M. Das and F. C. MacKintosh, Phys. Rev. E 84, 061906 (2011).

[35] L. Zhang, S. P. Lake, V. H. Barocas, M. S. Shephard, and R. C. Picu, Soft Matter 9, 6398 (2013).

[36] R. Rens, C. Villarroel, G. Düring, and E. Lerner, Phys. Rev. E 98, 062411 (2018).

[37] J. M. van Doorn, L. Lageschaar, J. Sprakel, and J. van der Gucht, Phys. Rev. E 95, 042503 (2017).

[38] Z. Xia and J. W. Hutchinson, J. Mech. Phys. Solids 48, 1107 (2000).

[39] A. P. Fantilli, H. Mihashi, and P. Vallini, Cem. Concr. Res. 39, 1217 (2009).

[40] I. Novak and L. Truskinovsky, Philos. Trans. R. Soc., A 375 (2017).

[41] A. Vainchtein, T. J. Healey, and P. Rosakis, Comput. Methods Appl. Mech. Eng. 170, 407 (1999).

[42] X. Ren and L. Truskinovsky, J. Elast. 59, 319 (2000).

[43] E. J. Doedel, T. F. Fairgrieve, B. Sandstede, A. R. Champneys, Y. A. Kuznetsov, and X. Wang, indy.cs.concordia.ca/auto/, 2007. 